Physics

Physics Research Publications

Resolving doppler-factor crisis in active galactic nuclei: Non-steady magnetized outflows

\author{
M. Lyutikov M. Lister
}

This paper is posted at Purdue e-Pubs.

http://docs.lib.purdue.edu/physics_articles/1297 


\title{
RESOLVING DOPPLER-FACTOR CRISIS IN ACTIVE GALACTIC NUCLEI: NON-STEADY MAGNETIZED OUTFLOWS
}

\author{
MaXim LyUtikov AND MATTHEW LisTer \\ Department of Physics, Purdue University, 525 Northwestern Avenue, West Lafayette, IN 47907-2036, USA \\ Received 2010 April 14; accepted 2010 July 9; published 2010 September 17
}

\begin{abstract}
Magnetically driven non-stationary acceleration of jets in active galactic nuclei results in the leading parts of the flow being accelerated to much higher Lorentz factors than in the case of steady-state acceleration with the same parameters. The higher Doppler-boosted parts of the flow may dominate the high-energy emission of blazar jets. We suggest that highly variable $\mathrm{GeV}$ and $\mathrm{TeV}$ emission in blazars is produced by the faster moving leading edges of highly magnetized non-stationary ejection blobs, while the radio data trace the slower-moving bulk flow. Thus, the radio and gamma-ray emission regions have different, but correlated, Doppler factors. High-energy emission is generated, typically within the optically thick core, in the outer parts of the broad-line emission region, avoiding the radiative drag on the faster parts of the flow. The radio emission should correlate with the gamma-ray emission, delayed with frequency-dependent time lag of the order of weeks to months. Model predictions compare favorably with the latest Fermi $\gamma$-ray and MOJAVE radio very long baseline interferometry results.
\end{abstract}

Key words: galaxies: active - galaxies: jets - gamma rays: general

Online-only material: color figures

\section{INTRODUCTION}

\subsection{Bulk Lorentz Factor Crisis in AGNs}

One of the defining characteristics of many active galactic nuclei (AGNs) is their flux variability in all spectral bands (Krolik 1999). In particular, extremely short timescales of $\mathrm{TeV}$ variability are challenging to the models. The rapid flares reported for Mrk 501 and PKS 2155-304, on timescales of 3-5 minutes (Albert et al. 2007; Aharonian et al. 2007), imply an emitting size smaller than the gravitational radius $t_{\mathrm{lc}} \sim$ hours of the supermassive black holes (BHs) of these blazars. There are two contradictory issues related to short timescale variability. First, it implies a small emission size, which poses a problem for efficiency of energy conversion into radiation. Second, there is the compactness problem (Guilbert et al. 1983). If variability is detected in $\gamma$-ray photons of energies exceeding the electron rest-mass energy, then the emission region contains photons which can pair produce. If the number density of these photons is too high, then none of the photons will escape the region. The solution to both problems is bulk relativistic motion toward the observer, which reduces the intrinsic luminosity, decreases the implied energy of the photons, and increases the internal timescales. The required Doppler factor then exceeds $\delta \geqslant 100$.

While highly relativistic motion may appear to be a cure-all, in AGNs the bulk Lorentz factor $\gamma$ can be directly constrained by very long baseline interferometry (VLBI) observations of bright blobs moving with apparent speeds on the sky, $\beta_{\text {app }}$, that appear to be superluminal. This type of motion occurs when the emitting region is moving relativistically and close to the line of sight (Rees 1966). The apparent transverse motion can exceed $c$ due to propagation effects. If a blob is moving along with the bulk flow of a jet and its velocity vector makes an angle, $\theta_{\mathrm{ob}}$, with the line of sight, then its apparent motion transverse to the line of sight will be $\beta_{\mathrm{app}}=\beta \sin \theta_{\mathrm{ob}} /\left(1-\beta \cos \theta_{\mathrm{ob}}\right)$. The maximum $\beta_{\text {app }}$ can reach is $\beta \gamma$ when $\theta_{\mathrm{ob}} \cong 1 / \gamma$. Thus, if the blob motion corresponds to the underlying bulk motion of the jet, measuring $\beta_{\text {app }}$ can constrain the possible bulk Lorentz factor, $\gamma$.

\subsection{MOJAVE Results}

The latest MOJAVE VLBI results do support the interpretation of moving jet features as physical entities, as opposed to patterns (Lister et al. 2009b). Observations of bidirectional motions, the near-absence of inward moving features, ejections of multiple blobs in the same jet with the same speed, and tight correlations of jet speeds with other properties, such as $\gamma$-ray emission, apparent $\gamma$-ray luminosity, brightness temperature, and even optical classification, all support the notion that the blob motion reflects the underlying flow.

The MOJAVE survey of compact, highly beamed radio-loud AGNs has analyzed the motion of emitting blobs in 127 jets and found that the observed superluminal speed distribution peaks at $\beta_{\text {app }} \sim 10$ and tapers off at $\beta_{\text {app }} \sim 50$ (Lister et al. 2009 b). This suggests that the bulk Lorentz factors of such objects are typically around $\sim 10$ and extend up to $\sim 50$, making the estimated values of $\gamma \geqslant 50$ for PKS 2155-304 and Mrk 501 rather difficult to reconcile with the radio data. Furthermore, direct VLBI observations of these sources on parsec scales have not even detected superluminal motion (Piner \& Edwards 2004; Giroletti et al. 2004).

VLBI observations of blazars such as PKS 2155-304 and Mrk 501 are not the only data which imply a low $\gamma$. Another way of investigating blazars is to search for their AGN counterparts whose jets are not directed along the line of sight, and which are presumed to be radio galaxies (there are actually two distinct types of radio galaxies, FR I and FR II, that are thought to correspond to the two categories of blazars, BL Lac objects and optically violent variable quasars; Urry \& Padovani 1995). $\mathrm{t}$, studies comparing the relative fluxes and numbers of radio galaxies and blazars point toward Lorentz factors of $\gamma<10$ (Henri \& Saugé 2006). Indeed, preliminary results of MOJAVE observations of low-luminosity jets from radio galaxies imply speeds of $c$ or less. Thus, there is an apparent contradiction between measured superluminal velocities and the bulk Lorentz factors required by radiation modeling. This is known as the "Blazars' Bulk Lorentz Factor Crisis" (Henri \& Saugé 2006). 
Multi-epoch very long baseline array (VLBA) observations of blazars at 15 and $43 \mathrm{GHz}$ (Lister et al. 2009c; Marscher $\&$ Jorstad 2010) have provided strong evidence that the $\gamma$-ray emission comes from the parsec-scale jet itself. This conclusion comes from (1) the strong association of 1FGL sources with flatspectrum radio sources, as opposed to lobe-dominated steepspectrum radio galaxies (Abdo et al. 2010b), (2) the correlation of $\gamma$-ray detection with higher radioactivity states (the parsecscale jet component is the one that varies in the radio; Kovalev 2009), (3) the better correlation seen between $\gamma$-ray flux versus VLBI compact radio flux, as compared to $\gamma$-ray flux versus total VLA flux (Kovalev 2009), and (4) the observed correlations between $43 \mathrm{GHz}$ VLBA core flux and $\gamma$-ray flare events in some individual blazars (Marscher \& Jorstad 2010; Marscher et al. 2010).

A natural explanation of the crisis is that radio and $\gamma$-ray emitting plasma are produced in different parts of the flow. Emitting regions can be either spatially separated (e.g., fast spin slow sheath) or temporally separated: Georganopoulos \& Kazanas (2003) suggested that flows experience quick bulk deceleration. Qualitatively, in our model, the emitting plasma is spatially separated, with the leading edge of an ejection accelerating faster than the bulk.

\section{NON-STATIONARITY IN AGN FLOWS}

Following Blandford \& Rees (1974), models of AGN jets typically assume steady-state injection conditions. This is based on the fact that the sonic time over the $\mathrm{BH}$ horizon is typically shorter than any observed timescales of variability of $\mathrm{BH}$ systems (except for such subtle effects as quasi-periodic oscillations in Galactic binaries, e.g., van der Klis 2005). By consequence, it is argued, a system reaches a quasi-equilibrium state. On the other hand, both Galactic $\mathrm{BH}$ candidates as well as AGN jets show a wide variety of non-stationary behavior. This non-stationarity is driven by various disk instabilities, occurring on the viscous timescale of the (inner) accretion disk.

From non-relativistic hydrodynamics, it is well known that the maximum velocity of stationary flows is typically smaller than those of non-stationary flows (for non-relativistic flows, this is true for adiabatic index less than three, e.g., Stanyukovich 1955). In the relativistic case, this translates into different fourvelocities. By the nature of the relativistic velocity transformations, the resulting maximal bulk Lorentz factors for nonstationary flows can be much higher than those of the stationary flow.

The efficiency of the BH-powered jet depends (e.g., in a Blandford \& Znajek (1977) paradigm of jet launching) on both the parameters of the BH (mass and spin) as well as on the magnetic field supplied by the disk. In addition, as we argue in this paper, jet acceleration may proceed more efficiently in the case of non-stationary outflows (see also Lyutikov 2010; Granot et al. 2010).

One expects that accretion onto the $\mathrm{BH}$ may change on a timescale of the order of the viscous timescale of the inner part of the disk, which may be weeks to months to years for a typical AGN (e.g., if we assume blob ejection timescales as being due to the inner disk instability). As the relativistic jet propagates away from the central $\mathrm{BH}$, it expands sideways, reaching a transverse scale of a fraction of a parsec at the distance of the order of a parsec. Close to the $\mathrm{BH}$, the corona of the accretion disk is hot, with a nearly relativistic speed of sound: the sonic timescale of the magnetosphere across the $\mathrm{BH}$ is much shorter than the viscous timescale of the inner accretion disk. Further out, the variability timescale of the jet will be shorter than the dynamical timescale of the corona across the jet. Observationally, the connection of the disk and jet activities is well established in the case of Galactic microquasars (Mirabel \& Rodríguez 1999). In AGNs, the data are controversial (Chatterjee et al. 2009).

Let us assume that a jet with an opening angle of $\Theta_{j} \sim 0.1$ is propagating through a corona, which is in hydrostatic equilibrium with the local sound speed $c_{s}$ close to the virial velocity, $c_{s}=\sqrt{G M_{\mathrm{BH}} / r}$. Relating the disk variability timescale to the Keplerian period near the $\mathrm{BH}, t_{d}=\xi r_{\mathrm{BH}} / c \approx 10$ days $M_{\odot, 9} \xi_{2}$, $\xi \gg 1$, where the sound crossing time across the jet is longer than the variability timescale for

$$
r_{\text {breakout }} \geqslant\left(\frac{\xi}{\Theta_{j}}\right)^{2 / 3} r_{\mathrm{BH}}=2 \times 10^{16} M_{\odot, 9} \xi_{2}^{2 / 3} \Theta_{j,-1}^{-2 / 3} \mathrm{~cm},
$$

where the notation $X_{a}$ implies $\left(X / 10^{a}\right)$ (e.g., $\xi_{2}=\xi / 100$ and $\Theta_{j,-1}=\Theta_{j} / 0.1$ ). At distances larger than Equation (1), the jet variability on a timescale of a hundred Keplerian periods near the $\mathrm{BH}, \xi_{2}=1$ proceeds on timescales shorter than the dynamical timescale across the jet, so the external medium does not have time to react to changing jet conditions. It is also required that the jet travel time to $r_{\text {breakout }}$ be smaller than the sound crossing time at $r_{\text {breakout }}$. (Otherwise, the low-density interface will be reached at distances where the jet travel time equals the variability timescale, $r \sim \xi r_{\mathrm{BH}}$.) This requires $\xi<1 / \Theta_{j}^{2}$.

A newly created jet will then propagate along a nearly empty channel, cleared by the previous jet activity. Thus, we expect that initially, close to the $\mathrm{BH}$, the non-stationary injected jet propagates through a relaxed corona and will have to "bore" its way through. After reaching the distance in Equation (1), the leading edge of the jet will break out into a low-density medium created by the previous jet activity. What are the consequences of this non-stationary behavior for jet acceleration?

As a model problem, we assume that a period of accretion lasting for a time $t_{\mathrm{d}}$ has brought onto the $\mathrm{BH}$ the magnetic field, initiating the Blandford-Znajek process. Since the external medium has a fairly low density, the jet expansion is relativistic (see Equation (7)). In a time $t_{\mathrm{d}}$, the jet inflates a bubble of linear size $c t_{\mathrm{d}} \sim r_{\text {breakout }}$. At a distance $r_{\text {breakout }}$, where the sonic time through the magnetosphere is of the order of the jet variability timescale, the newly inflated bubble reaches a near vacuum. What is the behavior of the jet starting at this point?

As a model problem, we considered (Lyutikov 2010) a onedimensional flow of cold magnetized plasma into a vacuum and into an external medium of density $\rho_{\mathrm{ex}}$. This is reviewed in Section 3. In Section 4, we apply the results to non-stationary outflows in AGNs.

\section{NON-STATIONARY RELATIVISTIC EXPANSION}

\subsection{Riemann Problem for Relativistic Expansion of Magnetized Gas}

Let us assume that before the breakout into the low-density medium the jet plasma is moving with velocity $\beta_{w}$ toward the external medium. We have found (Lyutikov 2010) an exact selfsimilar solution of the relativistic Riemann problem for the expansion of cold plasma with density $\rho_{0}$ and magnetic field $B_{0}$ (magnetization parameter $\sigma=B_{0}^{2} / \rho_{0}$; the magnetic field is normalized by $\sqrt{4 \pi}$ ), moving initially with velocity $v_{w}$ toward 

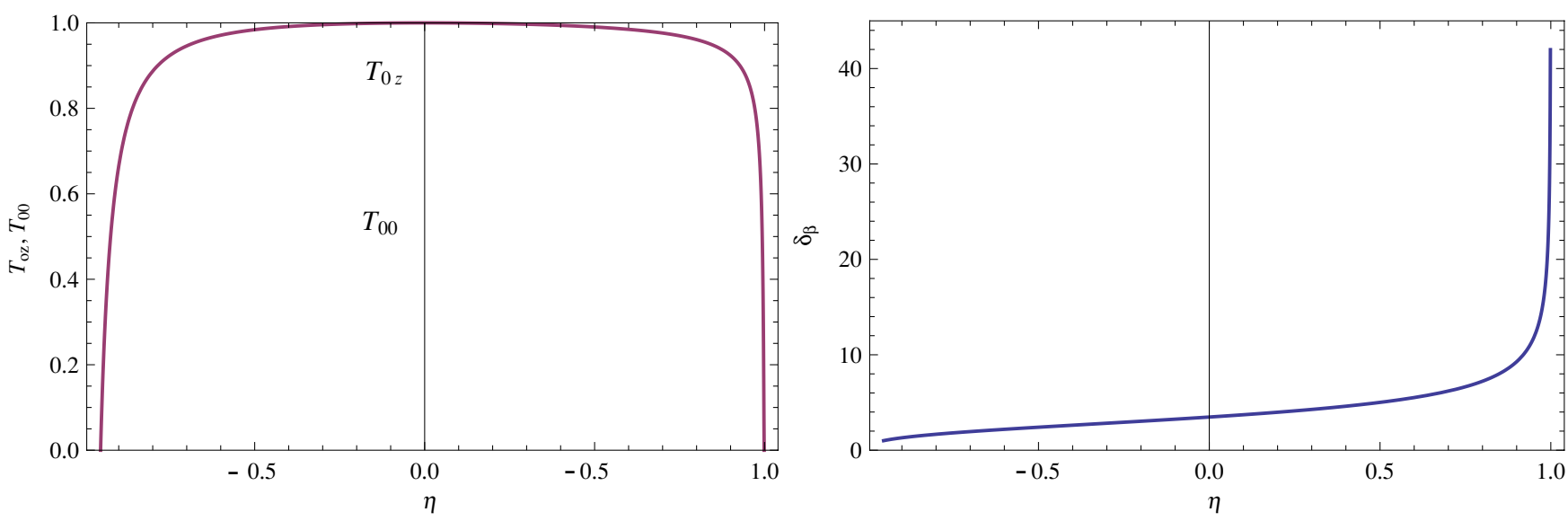

Figure 1. Total energy density $T_{00}$ and energy flux $T_{0 z}$ (left panel) and Doppler factor $\delta_{\beta}=\sqrt{(1+\beta) /(1-\beta)}$ (right panel) for self-similar expansion into a vacuum as functions of the self-similar coordinate $\eta=z / t$. Stationary initial conditions $\beta_{w}=0$ are assumed. The energy density is normalized to values in the undisturbed medium, while the energy flux is normalized to maximum values at $\eta=0$. Solutions extend from the front of the rarefaction wave at $\eta_{\mathrm{RW}}=-\sqrt{\sigma /(1+\sigma)}$ up to the vacuum interface $\eta_{\mathrm{vac}}=2 \frac{\sqrt{\sigma(1+\sigma)}}{(1+2 \sigma)}$. The Doppler factor is unity in the undisturbed plasma and reaches a value corresponding to $\gamma_{\mathrm{vac}}=1+2 \sigma$ at the vacuum interface. Plots are for $\sigma=10$.

(A color version of this figure is available in the online journal.)

the vacuum interface

$$
\begin{aligned}
& \delta_{\beta}=\delta_{\eta}^{2 / 3} \delta_{A, 0}^{2 / 3} \delta_{w}^{1 / 3}, \\
& \delta_{A}=\frac{\delta_{A, 0}^{2 / 3} \delta_{w}^{1 / 3}}{\delta_{\eta}^{1 / 3}},
\end{aligned}
$$

where the Doppler factors $\delta_{a}=\sqrt{\left(1+\beta_{a}\right) /\left(1-\beta_{a}\right)}$ are defined in terms of the plasma velocity $\beta$, local Alfvén velocity $\beta_{A}$, self-similar parameter $\eta=z / t$, initial wind velocity $\beta_{w}$, and the Alfvén velocity in the undisturbed plasma $\beta_{A, 0}$. The selfsimilar parameter $\delta_{\eta}$ ranges from the vacuum interface, where $\delta_{A}=1, \delta_{\eta, \max }=\delta_{A, 0}^{2} \delta_{w}$, and the front of the rarefaction wave, where $\delta_{\beta}=1, \delta_{\eta, \min }=1 /\left(\delta_{A, 0} \sqrt{\delta_{w}}\right)$. These equations give the velocity $\beta$, density $\rho=U_{A}^{2} \rho_{0} / \sigma$ (where $U_{A}=\beta_{A} / \sqrt{1-\beta_{A}^{2}}$ ), and proper magnetic field, $B=\left(\rho / \rho_{0}\right) B_{0}$, as a function of the self-similar variable $\eta=z / t$ (the expansion of plasma starts at $t=0, z=0$ and proceeds into a positive direction $z>0$ ). We stress that these solutions are exact; no assumptions about the value of the parameter $\sigma$ and velocity $v_{w}$ were made.

Particularly simple relations are obtained for plasma initially at rest expanding into vacuum $\beta_{w}=0, \delta_{\beta}=1$ (Lyutikov 2010). The flow accelerates from rest toward the vacuum interface (Figure 1). The bulk of the flow is moving with the Lorentz factor $\gamma^{\prime} \sim \sigma^{1 / 3}$. The flow becomes supersonic at $\eta=0$, at which point $\gamma^{\prime}=(\sigma / 2)^{1 / 3}$. The vacuum interface moves with the Lorentz factor $\gamma_{\text {vac }}^{\prime}=1+2 \sigma$. In the observer frame the vacuum interface is moving with $\delta_{\eta}=\delta_{A, 0}^{2} \delta_{w}$, which in the limit $\sigma, \gamma_{w} \gg 1$ gives

$$
\gamma_{\mathrm{vac}}=4 \gamma_{w} \sigma .
$$

As the flow expands, the energy density $T_{00}$ and the energy flux $T_{0 z}$ stay nearly constant in the bulk of the flow at a value $\approx B_{0}^{2} / 4$. The energy flux is maximal at the sonic point $\eta=0$, while the energy density slowly decreases toward the vacuum interface (Figure 1).

Most importantly, in a narrow region near $\eta_{\mathrm{vac}}=$ $2 \sqrt{\sigma(1+\sigma)} /(1+2 \sigma) \approx 1-1 /\left(8 \sigma^{2}\right)$, the Doppler factor increases from $\sim \sigma^{1 / 3}$ in the bulk to $\delta_{\beta, \max } \sim 4 \sigma$ on the vacuum interface. Values of $\delta_{\beta}>(1 / 2) \delta_{\beta, \max }$ are reached within a range of $\Delta \eta=7 /\left(8 \sigma^{2}\right)$ near the vacuum interface. The relative amount of energy with Doppler factors $\delta_{\beta}>(1 / 2) \delta_{\beta, \max }$ is $\sim 3 / \sigma^{2}$ (the total energy of the outflow at time $t$ is $E_{\text {tot }}=$ $\left.\rho_{0}(1+\sigma / 2) \sqrt{\frac{\sigma}{1+\sigma}} t \approx B_{0}^{2} t / 2\right)$. In addition, in a non-self-similar regime, most of the plasma will reach the vacuum terminal velocity, since the forward characteristics never cross the vacuum interface regardless of the dimensionality of the flow (Greenspan \& Butler 1962; Zeldovich \& Raizer 2003; see also Lyutikov 2010).

As the flow expands, the local magnetization

$$
\sigma_{\mathrm{loc}}=\frac{B^{2}}{\rho}\left(\frac{\delta_{A, 0}^{2 / 3}}{\delta_{\eta}^{1 / 3}}-\frac{\delta_{\eta}^{1 / 3}}{\delta_{A, 0}^{2 / 3}}\right)
$$

decreases (Figure 2). At the sonic point, $\sigma_{\mathrm{loc}}=(\sigma / 2)^{2 / 3}$.

\subsection{Expansion into Medium}

At small radii, the newly created jet has to bore through the dense corona. After the breakout, the jet will propagate into a low-density channel. In this section, we outline the jet dynamics before the breakout and estimate the effects of the residual density after the breakout.

Generally, if there is an outside medium with density $\rho_{\mathrm{ex}}$, we may identify two expansion regimes with different properties of the forward shock. For relativistically strong forward shocks, so that the post-shock pressure is much larger than the density, the Lorentz factor of the contact discontinuity (CD) is

$$
\gamma_{w} \approx\left(\frac{L}{\rho_{\mathrm{ex}} c^{3}}\right)^{1 / 4} r^{-1 / 2} .
$$

Relation (5) is a pressure balance on the $\mathrm{CD}$ of the wind luminosity $\sim L /\left(r^{2} c \gamma_{w}^{2}\right)$ and the ram pressure, $\sim \rho_{\mathrm{ex}} c^{2} \gamma_{w}^{2}$. Before the breakout, Equation (5) gives the motion of the newly created jet (its working surface) as a function of the external density (density of the corona $\rho_{\text {cor }}$ ) and jet power.

After the breakout, two expansion regimes are possible, corresponding to weak and strong forward shock. (Recall that 

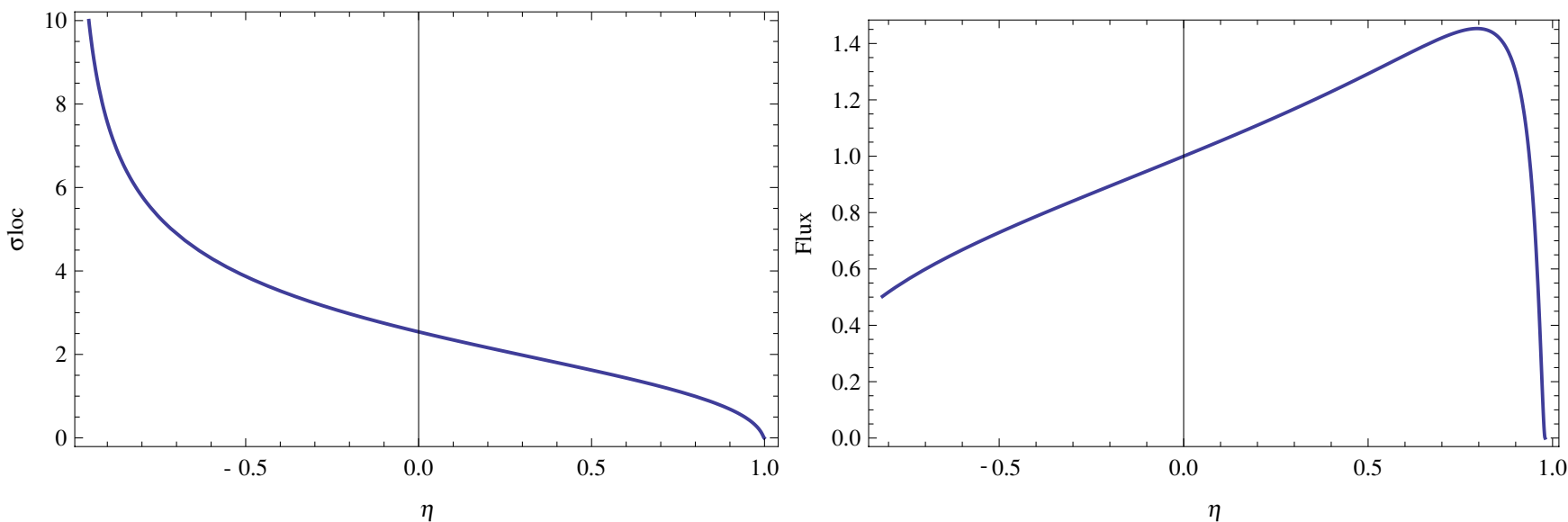

Figure 2. Left panel: local magnetization $\sigma_{\mathrm{loc}}$ as given by Equation (4). Initially, $\sigma=10$. Right panel: observed flux produced by the plasma expansion into vacuum, parameterized as a product of the rest-frame energy density and the Doppler factor cubed. The scale is normalized to the value at $\eta=0$.

(A color version of this figure is available in the online journal.)

we define weak/strong shocks by the post-shock temperature; it is relativistic for strong forward shocks.) For strong shocks, the Lorentz factor of the contact after breakout is determined by Equation (5), with $\rho_{\mathrm{ex}} \rightarrow \rho_{\text {res }}$, where $\rho_{\text {res }}$ is the residual density in the channel.

For weak forward shocks, the velocity of the $\mathrm{CD}$ approaches the expansion velocity into vacuum $\gamma_{\text {vac }}$ (Equation (3)). The transition between the strong and weak shocks, when $\gamma_{\mathrm{vac}} \sim \gamma_{w}$, occurs for

$$
\sigma_{\text {crit }} \sim\left(\frac{\rho_{\text {cor }}}{\rho_{\text {res }}}\right)^{1 / 4} .
$$

For $\sigma<\sigma_{\text {crit }}$, the forward shock is weak and the jet working surface propagates nearly as it does into vacuum.

\section{IMPLICATIONS FOR NON-STATIONARY ACCELERATION IN AGNs}

We propose that flares in $\mathrm{TeV}$ and $\mathrm{GeV}$ emitting blazars are produced in the leading expansion edges of non-stationary ejection events, moving with $\gamma_{\max } \geqslant 100$, while the observed velocities of the radio blobs correspond to the bulk motion with $\gamma_{\text {bulk }} \sim 10-50$. We expect that even relatively small amounts of energy dissipated in the fastest moving parts of the flow will be Lorentz boosted to high observed fluxes and highest energies. In this section, we discuss observational implications of the model.

In our picture, after the switch-on of the acceleration process, the AGN central engine produces a relativistic jet, and as the latter bores through the static corona, it can reach a relativistic Lorentz factor $\gamma_{w}$. The value of $\gamma_{w}$ can be determined by the pressure balance (Equation (5)) with a high external density. At the breakout radius (Equation (1)), the jet propagates with

$$
\gamma_{w}=\left(\frac{L}{\rho_{\text {cor }} c^{3}}\right)^{1 / 4} r_{\text {breakout }}^{-1 / 2}=20 L_{j, 46}^{1 / 4} \Theta_{j,-1} n_{\text {cor }}^{-1 / 4},
$$

where we have assumed $\xi=1 / \Theta_{j}^{2}$ and typical parameters of AGN outflows; $n_{\text {cor }}$ is the number density of particles in the corona.

After breakout, at $r>r_{\text {break }}$, the total Lorentz factor in the observer frame will be $2 \sigma^{1 / 3}$ times larger for the bulk flow, $\sim 2 \gamma_{w} \sigma^{1 / 3}$, and $4 \sigma$ times larger for the leading edge, $\sim 4 \gamma_{w} \sigma:$

$$
\begin{aligned}
\gamma_{\text {bulk }} \sim 2 \gamma_{w} \sigma^{1 / 3} & =40 \sigma^{1 / 3} L_{j, 46}^{1 / 4} \Theta_{j,-1} n_{\mathrm{cor}}^{-1 / 4}, \\
\gamma_{\mathrm{vac}} \sim 4 \gamma_{w} \sigma & =80 \sigma L_{j, 46}^{1 / 4} \Theta_{j,-1} n_{\mathrm{cor}}^{-1 / 4} .
\end{aligned}
$$

For values of $\sigma$ exceeding unity, the difference between Lorentz factors of the bulk flow and that of the leading edge is even greater.

Soon after the breakout, in the self-similar stage, the relative amount of energy in the fast leading tail is fairly small in the case of highly magnetized jets, $\sim 3 / \sigma^{2}$, yet it may dominate the beamed high-energy emission due to high Doppler boosting. For example, if we parameterize the observed intensity produced by the jet as a product of the rest-frame energy density, $T_{0 z} /\left(\gamma^{2} \beta\right)$, and Doppler factor cubed $\delta_{\beta}^{3}$, it will be dominated by the fast moving parts of the flow (Figure 2).

In addition, at later stages of expansion of a finite-size blob, a larger fraction of the material may be accelerated to the maximal Lorentz factor. If the initial blob had a size $L$ in its rest frame, most of the blob's material gets accelerated to the Lorentz factor $\gamma_{\text {bulk }}^{\prime} \sim 1+2 \sigma$ in time $\Delta t \sim 8 \sigma^{2} L / c$ (Lyutikov 2010). For a blob moving with $\gamma_{w}$ the acceleration time in the observer frame is further extended to $\Delta t \sim 16 \sigma^{2} \gamma_{w} L / c$. This time is, typically, longer than the timescale for blob interaction, Equation (13). Thus, the bulk of the material does not get accelerated to the maximum Lorentz factor $\gamma_{\max }$.

There are a number of correlations that we would expect in the model. First of all, we expect some correlation between the $\gamma$-ray and radio core fluxes, and this has already been seen by Fermi and MOJAVE (Kovalev 2009).

Since the $\gamma$-rays are produced in a faster moving part of the flow, we expect that the jets of $\gamma$-ray-selected AGNs are more aligned than those in radio-selected samples. This effect should be reflected in a flux-flux plot of a well-defined flux-limited blazar sample, due to the different degrees of Doppler beaming in the radio versus $\gamma$-ray regimes. For example, a tight linear correlation of intrinsic (i.e., unbeamed) radio versus $\gamma$-ray jet luminosity will be greatly smeared out in the flux-flux plot, although an upper envelope will still be present (see, e.g., simulations by Lister 2007). Indeed, the form and scatter of the observed radio- $\gamma$-ray correlation in blazars (Kovalev 2009) does support the notion that the $\gamma$-ray emission is likely boosted by a higher Doppler 
factor than the radio emission. This is also reflected in the superluminal speeds and apparent jet opening angles, which suggest higher Doppler factors and smaller viewing angles for Fermidetected AGNs (Lister et al. 2009a; Pushkarev et al. 2009).

On the other hand, a radio- $\gamma$-correlation crucially depends on the fact that the cores of AGN jets are optically thick to synchrotron emission up to the frequency-dependent radius (Blandford \& Konigl 1979, their Equation (28))

$$
r_{\text {core }} \approx 0.14 \mathrm{pc} \zeta_{R}^{2 / 3} L_{46}^{2 / 3} \gamma_{w, 1}^{-1 / 3} v_{10}^{-1},
$$

where $\zeta_{R}$ parameterizes the observed radio luminosity in terms of the total jet power $L_{R}=\zeta_{R} L$, with $\zeta_{R} \sim 0.01$; we also assumed here (and in the estimates below) that $\Theta_{j} \sim 1 / \gamma_{w}$, $\xi=1 / \Theta_{j}^{2}$. The core radius (9) is typically larger than the breakout radius (1). Thus, typically, the jet breakout will occur while the jet is still optically thick in the radio. In this case, the high-energy emission will not be accompanied by the simultaneous increase of radio flux (at least at low frequencies). At sufficiently high radio frequencies, the radius where the jet becomes optically thin (Equation (9)) may become comparable to the breakout radius (Equation (1)).

The most generic prediction of the model is that $\gamma$-ray event should precede the radio blob ejection by

$$
\begin{aligned}
\Delta t_{\gamma-R}= & \left(r_{\text {core }} / c\right)\left(1-\beta \cos \theta_{\mathrm{ob}}\right) \sim \frac{r_{\text {core }} / c}{2 \gamma_{w}^{2}} \\
& \sim 8 \zeta_{R}^{2 / 3} L_{46}^{2 / 3} \gamma_{w}^{-7 / 3} v_{9}^{-1} \text { days. }
\end{aligned}
$$

Recently, Pushkarev et al. (2010; see also Marscher et al. 2010; Mahony et al. 2010) reported the detection of a non-zero time delay between radio emission measured by the VLBA at $15.4 \mathrm{GHz}$ and gamma-ray radiation (gamma-ray leads radio) seen by Fermi. This strongly indicates that the gamma-ray emission is generated within the compact region of the $15 \mathrm{GHz}$ VLBA core.

Also, since this timescale (Equation (10)) is fairly long, we expect occasional $\gamma$-ray flares without radio blob ejection; this appears to be the case in $\mathrm{TeV}$ flares associated with high-energypeaked AGN such as Mrk 421 and Mrk 501, in which no major changes in VLBI radio structure are seen (MOJAVE program; E. Ros et al. 2010, in preparation). Furthermore, the $\gamma$-ray events should be better correlated with radio blob ejection at high radio frequencies. Higher resolution monitoring of MOJAVE sources by the Boston University group (Jorstad et al. 2005) at $43 \mathrm{GHz}$ has revealed instances of jet features moving at higher speeds than those seen at $15 \mathrm{GHz}$ (Lister et al. 2009b) that fade out very rapidly close to the core region. The extensive VLBA+Fermi data currently being gathered on these AGNs should provide a useful test of this prediction.

The above correlations assume that the $\gamma$-ray photons produced at the breakout radius (1) do not suffer from absorption. This depends on the compactness parameter corresponding to the bulk motion of the jet (since $\gamma$-ray photons can pair produce on a lower moving bulk plasma):

$$
l \sim \frac{1}{\gamma_{w}^{6}} \frac{\sigma_{T}}{m_{e} c^{3}} \frac{L_{\gamma}}{r_{\text {breakout }}}=10^{-7} L_{\gamma, 44} \Theta_{j,-1}^{2} \gamma_{w, 1}^{-6} L_{44} M_{9}^{-1}
$$

$\left(\xi=1 / \Theta_{j}^{2}\right.$ is assumed here and below). On the other hand, the $\gamma$-ray variability timescale depends on the Lorentz factor of the leading edge:

$$
\Delta t \sim \frac{r_{\text {breakout }} / c}{2 \gamma_{\text {vac }}^{2}}=\frac{r_{\text {breakout }} / c}{32 \gamma_{w}^{2} \sigma^{2}}=150 \sec \sigma^{-2} \Theta_{j,-1}^{-2} M_{9} .
$$

Thus, the model is able to accommodate both the requirement of small optical depth for $\gamma$-ray photons and the short timescale variability, down to a few minutes.

The jets of lower power FR I sources and higher power FR II sources have somewhat different morphology on mas scales. Relatively low-power blazar jets in the MOJAVE program like Mrk 421 or Mrk 501 show a very smooth fall-off in radio intensity with distance from the core, and have maintained this structure over more than a decade of VLBI monitoring. In contrast, higher power blazars such as 3C 279 display jet morphologies dominated by individual bright knots that continually emerge from the core and move downstream at superluminal speeds (see Figure 3).

One possible explanation for the dichotomy of jet properties is that the emitted blobs merge, creating smooth large-scale profiles. If the blobs are ejected on a timescale $t_{d}=\xi r_{\mathrm{BH}} / c$, they will merge at distance (in the observer frame the time of merger is determined by the Lorentz factor of the trailing edge of the preceding injection $\gamma \sim \gamma_{w} /(4 \sigma)$ :

$$
r_{\text {merge }} \sim 2 c t_{d}\left(\frac{\gamma_{w}^{2}}{16 \sigma^{2}}\right) \approx 3 \times 10^{20} \mathrm{~cm} \frac{1}{\sigma^{2}} \Theta_{j,-1}^{-2} .
$$

Thus, at linear scales $\geqslant 100 \mathrm{pc}$, the jet is expected to be mostly smooth. Note that since in blazars the angle between the jet direction and the line of sight is small, the projected distance corresponding to Equation (13) is small. For example, with the jets in Mrk 421 or Mrk 501 at $z=0.03$ oriented at a few degrees to the line of sight, this corresponds to a few milliarcseconds projected on the sky. In addition, since the radius $r_{\text {merge }}$ depends sensitively on the assumed bulk Lorentz factor, our model predicts that in powerful FR II jets, the jet may remain knotty up to the kiloparsec scale.

Can the observed morphology of the jets be used to determine intrinsic jet properties, like bulk Lorentz factor and magnetization? High-frequency-peaked blazars (HBLs) are underrepresented in the MOJAVE sample; in fact, there are no HBLs in the complete radio-selected sample (Lister \& Homan 2005). On the other hand, HBLs are well represented in the Fermi sample (Abdo et al. 2010a). In the framework of our model, this can be due to their lower bulk Lorentz factors, but high magnetization (see Equation (10)). It would then imply relatively smaller merging distances (Equation (13)) and smoother jet structures, consistent with observations (Figure 3). An obvious caveat in this argument is that HBLs have flatter $\gamma$-ray spectra and are more likely to be seen by the Fermi LAT. Further careful analysis of beaming and instrumental selection effects is needed to explore these possibilities more fully.

FR I and FR II sources could also have different intrinsic variability timescales. In our present model, we relate the variability timescale to the mass of the central $\mathrm{BH}$, which does not vary considerably between FR I and FR II sources (Ghisellini \& Celotti 2001).

\section{DISCUSSION}

In this paper, we discuss the effects associated with nonstationarity of the jet ejection. In particular, we argue that the leading edge of a non-stationary magnetized outflow can achieve a bulk Lorentz factor much larger than would be inferred for a steady-state outflow given similar conditions. In the case of the expansion of a highly magnetized plasma, the ratio of Lorentz factors of the bulk flow and that of the leading edge can be as high as $2 \sigma^{2 / 3}$ ( $\sigma$ is plasma magnetization). This ratio can reach 

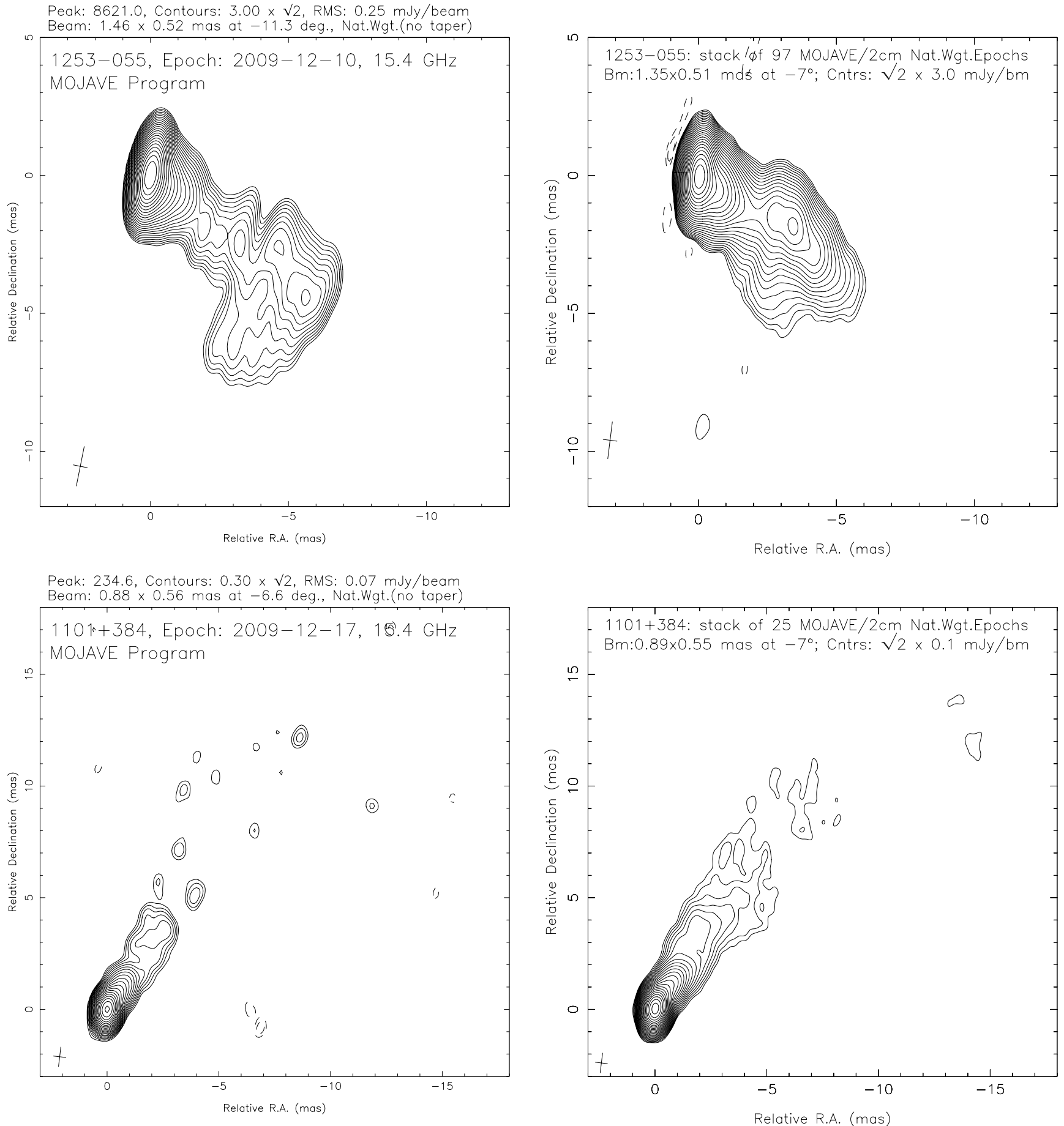

Figure 3. MOJAVE $15 \mathrm{GHz}$ VLBA images of low-energy-peaked quasar 3C 279 (1253-055; top two panels) and high-energy-peaked BL Lac object Mrk 421 $(1101+384$; bottom two panels). The left-hand panels show the parsec-scale jet structure in a recent MOJAVE epoch. The right-hand panels show time-averaged images created by combining archival VLBA epochs from 1995 to 2009. The relatively smooth fall-off in jet intensity downstream from the bright core in Mrk 421 is a characteristic of high-energy-peaked jets in the MOJAVE sample. These jets display very few changes in their radio jet structure over time, as indicated by the similarity in the inner 5 mas of the single epoch and stacked images of Mrk 421. By contrast, the jets of flat-spectrum radio quasars such as 3C 279 generally display a constantly varying structure that is dominated by numerous bright superluminal knots (Lister et al. 2009b, 2009c).

into the tens for highly magnetized flows with $\sigma \sim 10$. We suggest that the Doppler-factor crisis in AGNs (a difference of the Doppler factors inferred from radiation modeling, especially of short timescale $\mathrm{TeV}$ flares and from the observations of radio blobs) may be resolved by non-stationary outflows: highly variable emission is produced by the fast-moving leading edge of an expansion, while the radio data trace the slower-moving bulk flow.
One of the main implications of the model is that the radio and $\gamma$-ray emitting plasma has different Lorentz factors. This explains, for example, the fact that, on the one hand, there is solid evidence that $\mathrm{GeV}$ emission comes from the jet, yet on the other hand some Fermi blazars do not show superluminal features at all (see Section 1.2).

This model places the acceleration region of the $\gamma$-ray emitting plasma at a substantial distance from the central $\mathrm{BH}$, 
hundreds to thousands of Schwarzschild radii, but still, typically, inside the optically thick radio core. These distances roughly correspond to the size of the broad-line emission region (BLR). The size of a BLR is $\sim 0.1 \mathrm{pc}$ in Seyfert 1 galaxies, and up to $1 \mathrm{pc}$ in FSRQs. It is expected then that the radiative drag on the leading edge should be smaller than in the case where acceleration occurs close to the $\mathrm{BH}$. Thus, the model may be able to avoid the radiative drag and the related problem of the observed absence of the bulk Comptonization spectral bump (Sikora et al. 1994).

The model compares favorably with observations. First of all, the predicted correlated $\gamma$-radio fluxes (Mahony et al. 2010) with a delay has been recently measured with, approximately, the correct value of time delay (Pushkarev et al. 2010), of the order of a month. The model postulates that $\gamma$-rays have higher Doppler boosting than radio, and thus have intrinsically shorter variability timescales. In radio, variability is also smoothed out by the fact that emission occurs in the optically thick regime.

At the present stage the model is oversimplified, especially in the treatment of the jet propagation through the corona. The disk corona is not in a state of a hydrostatic equilibrium, but generates a superfast (and probably relativistic) wind (Komissarov \& McKinney 2007). As a result, the magnetosphere cannot fill in the region within the core of the jet quickly enough. Thus, the evolution of the disk and the jet should be considered selfconsistently and in a time-dependent manner. For example, nonstationarity may be due to overloading the $\mathrm{BH}$ region with too much infalling mass on top, so that the jet and wind temporarily cannot escape due to the ram pressure of the infalling medium. As the jet-wind energy builds or the ram pressure subsides, eventually the jet escapes. In addition, as the wind will pass through the termination shock, the local sound speed will increase locally. Overall, the self-consistent account of the disk-jet-wind interaction is likely to produce more complicated time variability than the one described here.

The suggested model is qualitatively different from the internal shock models, where non-stationarity is invoked to produce shocks and dissipate the energy of the relative bulk motion of the colliding media. Collision of strongly magnetized plasma blobs results in only weakly dissipative internal shocks. The fast leading expansion edges will generate powerful shocks in the surrounding medium that may produce the high-energy emission. We do not address the question of how magnetic and bulk energy is converted into radiation.

A somewhat similar continuous acceleration mechanism was proposed by Tchekhovskoy et al. (2010) (see also Komissarov et al. 2010). It relies on sideways expansion of the jet after the breakout. Sideways expansion of unconfined magnetically dominated plasma proceeds with the Lorentz factor $1+2 \sigma$, so that the total Lorentz factor (of a plasma near the edge, affected by the rarefaction wave) is $(1+2 \sigma) \gamma_{w}$, two times smaller than for the case of an expansion wave propagating along the direction of motion. This factor of 2 may have an important effect on the escape of high-energy radiation, since the optical depth to pair production scales approximately as $\Gamma^{-6}$, a difference in a factor of 2 in $\Gamma$ will result in a difference of 64 in the optical depth. (Tchekhovskoy et al. (2010) cannot treat parallel acceleration akin to breaking into vacuum since the rotation of the central engine is turned on gradually for numerical reasons.)

In summary, magnetically driven non-stationary jet acceleration can provide a potential resolution of the longstanding bulk Lorentz factor crisis in blazars. The rich Fermi-VLBA data set that is currently being gathered on a broad set of AGNs should provide an excellent means of testing our proposed scenario.

This research has made use of data from the MOJAVE database that is maintained by the MOJAVE team. The MOJAVE program is supported under National Science Foundation grant 0807860-AST and NASA-Fermi grant NNX08AV67G. The National Radio Astronomy Observatory is a facility of the National Science Foundation operated under cooperative agreement by Associated Universities, Inc. We thank the organizers and participants of the Fermi meets Jansky workshop.

\section{REFERENCES}

Abdo, A. A., et al. 2010a, ApJ, 716, 30

Abdo, A. A., et al. 2010b, ApJS, 188, 405

Aharonian, F., et al. 2007, ApJ, 664, L71

Albert, J., et al. 2007, ApJ, 669, 862

Blandford, R. D., \& Konigl, A. 1979, ApJ, 232, 34

Blandford, R. D., \& Rees, M. J. 1974, MNRAS, 169, 395

Blandford, R. D., \& Znajek, R. L. 1977, MNRAS, 179, 433

Chatterjee, R., et al. 2009, ApJ, 704, 1689

Georganopoulos, M., \& Kazanas, D. 2003, ApJ, 594, L27

Ghisellini, G., \& Celotti, A. 2001, A\&A, 379, L1

Giroletti, M., et al. 2004, ApJ, 600, 127

Granot, J., Komissarov, S., \& Spitkovsky, A. 2010, arXiv:1004.0959

Greenspan, H. P., \& Butler, D. S. 1962, J. Fluid Mech., 13, 101

Guilbert, P. W., Fabian, A. C., \& Rees, M. J. 1983, MNRAS, 205, 593

Henri, G., \& Saugé, L. 2006, ApJ, 640, 185

Jorstad, S. G., et al. 2005, AJ, 130, 1418

Komissarov, S. S., \& McKinney, J. C. 2007, MNRAS, 377, L49

Komissarov, S. S., Vlahakis, N., \& Konigl, A. 2010, MNRAS, 407, 17

Kovalev, Y. Y. 2009, ApJ, 696, L17

Krolik, J. H. 1999, Active Galactic Nuclei: From the Central Black Hole to the Galactic Environment (Princeton, NJ: Princeton Univ. Press)

Lister, M. L. 2007, in AIP Conf. Ser. 921, The First GLAST Symposium, ed. S. Ritz, P. Michelson, \& C. A. Meegan (Melville, NY: AIP), 345

Lister, M. L., \& Homan, D. C. 2005, AJ, 130, 1389

Lister, M. L., Homan, D. C., Kadler, M., Kellermann, K. I., Kovalev, Y. Y., Ros, E., Savolainen, T., \& Zensus, J. A. 2009a, ApJ, 696, L22

Lister, M. L., et al. 2009b, AJ, 138, 1874

Lister, M. L., et al. 2009c, AJ, 137, 3718

Lyutikov, M. 2010, Phys. Rev. E, submitted

Mahony, E. K., Sadler, E. M., Murphy, T., Ekers, R. D., Edwards, P. G., \& Massardi, M. 2010, ApJ, 718, 587

Marscher, A. P., \& Jorstad, S. G. 2010, arXiv:1005.5551

Marscher, A. P., et al. 2010, ApJ, 710, L126

Mirabel, I. F., \& Rodríguez, L. F. 1999, ARA\&A, 37, 409

Piner, B. G., \& Edwards, P. G. 2004, ApJ, 600, 115

Pushkarev, A. B., Kovalev, Y. Y., \& Lister, M. L. 2010, arXiv:1006.1867

Pushkarev, A. B., Kovalev, Y. Y., Lister, M. L., \& Savolainen, T. 2009, A\&A, 507, L33

Rees, M. J. 1966, Nature, 211, 468

Sikora, M., Begelman, M. C., \& Rees, M. J. 1994, ApJ, 421, 153

Stanyukovich, K. 1955, Non-Stationary Motion in Continuous Medium (in Russian; Moscow: State Publ.)

Tchekhovskoy, A., Narayan, R., \& McKinney, J. C. 2010, ApJ, 711, 50

Urry, C. M., \& Padovani, P. 1995, PASP, 107, 803

van der Klis, M. 2005, Astron. Nachr., 326, 798

Zeldovich, Y. B., \& Raizer, Y. P. 2003, Physics of Shock Waves (New York: Dover) 